\title{
Multifaceted Approach to Personality Assessment in Cheetahs (Acinonyx jubatus)
}

\author{
Catherine B. Razal $^{1}$, Corrine B. Pisacane ${ }^{2}$, \& Lance J. Miller ${ }^{1^{*}}$
}

${ }^{1}$ Chicago Zoological Society - Brookfield Zoo

${ }^{2}$ San Diego Zoo Institute for Conservation Research

*Corresponding author (Email: Lance.Miller@CZS.org)

Citation - Razal, C. B., Pisacane, C. B., \& Miller, L. J. (2016). Multifaceted approach to personality assessment in cheetahs (Acinonyx jubatus). Animal Behavior and Cognition, 3(1), 22-31. doi: 10.12966/abc.02.02.2016

\begin{abstract}
The purpose of this study was to assess the personality of 17 South African cheetahs at the San Diego Zoo Safari Park. A multifaceted approach of observer ratings (rating method), behavioral data (coding method) and hormone monitoring was used to examine individual differences. Knowledge of these individual differences, i.e., personality, is essential to better husbandry and management in a zoological facility. Principal Components Analysis yielded five personality components: Insecure, Aggressive, Interactive, Active, and Unsociable. Comparing the personality components with gender, reproductive success, and hand-rearing history of the individuals revealed significant associations. The components Aggressive and Interactive had a positive correlation with behavioral diversity, and the component Unsociable had a positive correlation with self-maintenance. There was a significant gender difference, with males scoring higher on Aggressive and Interactive. Individuals who scored higher on these components also had higher levels of behavioral diversity, and males displayed higher levels of behavioral diversity than females. In addition, individuals that were reproductively successful scored higher on the component Unsociable, as well as displayed higher FGM levels. This study demonstrates the value of using a multifaceted approach to further understand and assess animal personality. Information gained from this study can ultimately help zoological facilities manage and assist with reproductive efforts in populations that are not currently self-sustaining, such as the cheetah.
\end{abstract}

Keywords - Cheetah, Acinonyx jubatus, Personality, Reproductive success, Animal welfare

Zoos and aquariums provide a great opportunity for researchers to observe species and examine individual behavioral differences (Watters \& Powell, 2011). These individual behavioral differences observed consistently over time and across situations are termed "personality" (Freeman \& Gosling, 2010; Gosling, 2001). Studies involving personality research in a zoological setting have gained popularity during the last decade, providing consistent examples that animals do exhibit individual behavioral traits. However, it was only until recently that zoological institutions realized the importance of utilizing personality as a tool towards understanding and promoting animal welfare and management (Powell \& Gartner, 2011).

Observer ratings have been used widely across personality research, since it consists of having those who know the animals most (i.e., keepers) score an animal's behavioral tendencies based on a provided list of traits or descriptions (Highfill, Hanbury, Kristiansen, Kuczaj, \& Watson, 2010). Observer ratings are a valuable tool for measuring individual behaviors, and can be beneficial once they produce 
reliable data (Meagher, 2009). Previous studies have shown that observer ratings can group behaviors that predict breeding success, as demonstrated in black rhinoceros (Diceros bicornis; Carlstead, Fraser, Bennett, \& Kleiman, 1999; Carlstead, Mellen, \& Kleiman, 1999) and cheetahs (Acinonyx jubatus;

Wielebnowski, 1999). Wielebnowski (1999) applied behavioral assessment to understand breeding success based on individual differences of cheetahs and found that individual behavioral variation, i.e., personality, can be reliably assessed in cheetahs using observer ratings. Wielebnowski (1999) found that non-breeders of both sexes scored higher on the personality component Tense-Fearful than breeders. This suggested that individuals with a higher level of fearfulness had less ability to cope and reproduce in managed care, and also implied that these individuals require more secluded areas in order to breed successfully. The results from this study are an example at how assessing personality can predict not only reproductive success, but also how individual responses to different situations can be used to aid management practices. Similarly, Gartner and Powell (2012) assessed personality in snow leopards by analyzing individuals' reactions to a novel-object, and discovered correlations between visiting the object and those with the personality traits (called dimensions in the study) of Active/Vigilant and Curious/Playful. These behaviors were indicative of individual variation, and could imply that bolder animals need more space and novel items to explore in their environment (Gartner \& Powell, 2012; Powell \& Gartner, 2011).

These studies suggest that zoos are using observer ratings to assist animal husbandry and improve management (Kuhar, Stoinski, Lukas, \& Maple, 2006; Meagher, 2009). However, observer ratings are not enough to provide a clear assessment of an individual, and must be used in conjunction with other methods (Meagher, 2009). Implementing a multifaceted approach that involves observer ratings alongside the coding method and hormone monitoring can provide greater knowledge on assessing personality. The coding method differs from the rating method of measuring personality by having observers score based on the animal's specific, naturally-occurring behaviors, such as eating or grooming (Vazire \& Gosling, 2004). Species with poor breeding success in zoological institutions are likely to benefit from a multifaceted approach assessing personality, such as the cheetah (Brown et al., 1996; Caro, 1993; Laurenson, Caro, \& Borner, 1992; Marker \& O’Brien, 1989; Marker-Kraus \& Kraus, 1997). In spite of Wielebnowski (1999), and other extensive breeding efforts at various zoos, the managed cheetah population is currently not self-sustaining (Marker, 2013).

The objectives of the current study were to apply a multifaceted method to assess cheetah personality. This included rating and coding the behavior of cheetahs, examining fecal glucocorticoid metabolite (FGM) profiles and comparing this information to the gender, reproductive success, and handrearing history of individuals at the San Diego Zoo Safari Park (SDZSP). Personality components were also compared to indicators of welfare. Information gained from this study can assist with the management of the cheetah population in zoological facilities.

\section{Method}

\section{Study Animals and Facilities}

The study subjects consisted of 17 South African cheetahs ( 8 males, 9 females) ranging from ages 1 to 12 years-old $\left(M_{\text {age }}=7.12\right.$ years), located at SDZSP in Escondido, California (Table 1). Reproductive success was defined as any cheetah producing at least one litter in their lifetime, based on a similar definition in the Wielebnowski (1999) study. Five out of the 17 cheetahs were deemed reproductively successful since these individuals had at least one litter ( 3 males, 2 females). Hand-reared individuals were those that were raised by keeper staff at SDZSP (4 females).

Fifteen cheetahs were housed at the cheetah breeding area (CBA), a facility towards the back of the property away from the visiting public, while two females were housed at an enclosure open to the public in SDZSP. The subjects at CBA were in adjacent, outdoor chain-link enclosures. The males occupied eight pens of varying sizes, the smallest measuring $230 \mathrm{~m}^{2}$, and the largest measuring approximately $12,150 \mathrm{~m}^{2}$; females inhabited six pens, the smallest being $190 \mathrm{~m}^{2}$, and the largest 
measuring 7,080 $\mathrm{m}^{2}$ (Table 1). Due to the proximity of the enclosures, most of the cheetahs had visual access of each other as no permanent visual barrier was established. The public enclosure housing the two females measured approximately $1,900 \mathrm{~m}^{2}$. All enclosures had natural grass and dirt substrate, and consisted of either various levels in the terrain or multiple trees and brush to offer equal opportunity of hiding places.

Table 1

\begin{tabular}{|c|c|c|c|c|c|}
\hline Name & Sex & Date of Birth & $\begin{array}{c}\text { Age } \\
\text { (At Start of Study) }\end{array}$ & Location & $\begin{array}{c}\text { Approximate Enclosure } \\
\text { Size }\left(\mathbf{m}^{2}\right)\end{array}$ \\
\hline C1 & $\mathrm{F}$ & $10 / 11 / 2003$ & 9 & Park, with C2 & 1900 \\
\hline $\mathrm{C2}$ & $\mathrm{F}$ & $10 / 11 / 2003$ & 9 & Park, with C1 & 1900 \\
\hline C3 & $\mathrm{F}$ & $6 / 27 / 2004$ & 8 & $\mathrm{CBA}$ & 1230 \\
\hline $\mathrm{C} 4$ & $\mathrm{~F}$ & $8 / 9 / 2005$ & 7 & CBA & 190 \\
\hline C5 & $\mathrm{F}$ & $8 / 9 / 2005$ & 7 & CBA & 780 \\
\hline C6 & $\mathrm{F}$ & $8 / 9 / 2005$ & 7 & CBA & 190 \\
\hline $\mathrm{C} 7$ & $\mathrm{~F}$ & $11 / 8 / 2008$ & 4 & CBA, with $\mathrm{C} 8$ & 780 \\
\hline C8 & $\mathrm{F}$ & $11 / 8 / 2008$ & 4 & CBA, with C7 & 780 \\
\hline C9 & $\mathrm{F}$ & $6 / 17 / 2009$ & 3 & $\mathrm{CBA}$ & 7080 \\
\hline C10 & $\mathrm{M}$ & $4 / 26 / 2000$ & 12 & CBA & 12150 \\
\hline C11 & M & $7 / 26 / 2001$ & 11 & CBA, with $\mathrm{C} 12$ & $230 ; 230^{\mathrm{a}}$ \\
\hline C12 & M & $7 / 26 / 2001$ & 11 & CBA, with C11 & $230 ; 230^{\mathrm{a}}$ \\
\hline C13 & M & $12 / 7 / 2003$ & 9 & CBA, with $\mathrm{C} 14,15$ & 4050 \\
\hline $\mathrm{C} 14$ & $\mathrm{M}$ & $12 / 7 / 2003$ & 9 & CBA, with $\mathrm{C} 13,15$ & 4050 \\
\hline C15 & $\mathrm{M}$ & $12 / 7 / 2003$ & 9 & CBA, with $\mathrm{C} 13,14$ & 4050 \\
\hline C16 & M & $5 / 28 / 2011$ & 1 & CBA, with $\mathrm{C} 17$ & $325 ; 650^{\mathrm{a}}$ \\
\hline C17 & M & $5 / 28 / 2011$ & 1 & CBA, with $\mathrm{C} 16$ & $325 ; 650^{\mathrm{a}}$ \\
\hline
\end{tabular}

${ }^{\mathrm{a}}$ Subject occupied two enclosures, both in $\mathrm{m}^{2}$.

\section{Behavioral Observations}

Two observations per cheetah were conducted each day, five days a week from March 4, 2013 to May 31, 2013. The order in which the subjects were observed and the time of day were determined by a randomized sequence using Microsoft Excel®. At CBA, observations were recorded directly in front of each enclosure, and at SDZSP, observations were conducted from the visitor pathway. Behavioral states were collected in 10 minute segments using instantaneous sampling at one minute intervals (Table 2). All observations were carried out by four trained volunteers, each using data sheets to record and stopwatches to determinate the intervals. Inter-observer reliability was tested over a two-week period in which all observers recorded multiple sessions simultaneously and independently. Observer reliability was assessed at the end of each day of the two-week period using percent agreement for the behavioral states $(80.0 \%)$ and Pearson's correlation coefficient for the behavioral events $(R>0.80)$.

\section{Radioimmunoassay (RIA) Procedure for FGM Analysis}

Collection. Fecal samples were collected daily by zookeepers from March 4, 2013 to May 31, 2013. For the subjects that were housed together, different colors of non-toxic glitter were added to the diet of those individuals to aid in identifying samples. Fecal samples were collected in $30 \mathrm{~mL}$ labelled medicine cups and frozen at $-20^{\circ} \mathrm{C}$. Samples were then lyophilized using a freeze dryer (Flexi-Dry, FTS Systems, Inc. Stone Ridge, NY) for 96 hrs. Once the samples were lyophilized, they were crushed, sifted, and $0.2 \mathrm{~g}$ of the fecal sample was weighed into a $16 \mathrm{X} 100 \mathrm{~mm}$ glass tube for steroid extraction. 
Extraction. A phosphate-saline buffer containing 50\% methanol, $0.1 \%$ bovine serum albumin and $0.05 \%$ Tween 20 (polyoxyethylene sorbitan monolaurate, a surfactant) was added to the samples to extract glucocorticoid metabolites. Samples were vortexed for $30 \mathrm{~s}$, and then placed on a rocker overnight $(16 \mathrm{hrs}$ at $400 \mathrm{rpm})$. The following day, the samples were taken off the rocker and allowed to settle for one hour, then the extract was pipetted into separate tubes and spun in a centrifuge for one hour at 4,000 $\mathrm{rpm}$. The extract was decanted into a second set of labeled tubes, capped and refrigerated at $-4^{\circ} \mathrm{C}$ if being assayed immediately or frozen at $-20^{\circ} \mathrm{C}$ until analysis.

Table 2

Ethogram of Behavioral States Observed During the Study

\begin{tabular}{ll}
\hline Behavior & Operational Definition \\
\hline Explore/Interact Enrichment & $\begin{array}{l}\text { Licking, sniffing, manipulating enrichment object (non-permanent) within the } \\
\text { exhibit. } \\
\text { Licking, sniffing, manipulating environment including urine/feces but not } \\
\text { including enrichment (non-permanent). }\end{array}$ \\
Explore/Interact Environment & Animal is engaging in groom self, urination, or defecation. \\
Self-Maintenance & Animal is engaged in grooming or affiliative contact with another individual. \\
Social Positive & $\begin{array}{l}\text { Animal is engaged in aggressive behavior (e.g., bite, paw swipe, etc.) including } \\
\text { chase if preceded or followed by another aggressive behavior. }\end{array}$ \\
Social Negative & $\begin{array}{l}\text { Animal is engaged in social (e.g., chase), solitary (e.g., rolling), or object play } \\
\text { (e.g., play with object) }\end{array}$ \\
Play & $\begin{array}{l}\text { Animal is engaging in a repetitive ambulatory movement after traversing the } \\
\text { same pathway at least twice. }\end{array}$ \\
Pacing & Animal is engaging in any other abnormal behavior besides pacing.
\end{tabular}

RIA procedure. FGM values were measured by $3 \mathrm{H}$ radioimmunoassay (RIA) using an antibody produced against corticosterone-3-carboxymethyloxime: BSA (ICN Biomedicals, Costa Mesa, CA). Samples were brought to room temperature and $20 \mu \mathrm{L}$ of sample was pipetted into labelled $12 \mathrm{X} 75 \mathrm{~mm}$ tubes for the assay in duplicate. Tritiated corticosterone $(10,000 \mathrm{cpm}$, PerkinElmer Life Sciences, Boston, MA, USA) was used in the assay to compete with the endogenous glucocorticoid metabolites. PBS with Bovine Serum Albumin (BSA 0.35\%), PBS without BSA, and tracer solution was distributed into all tubes. An antibody was added to necessary tubes, then the assay was vortexed and incubated overnight at $4^{\circ} \mathrm{C}$. After incubation, charcoal dextran solution $(250 \mu \mathrm{L})$ was added to terminate the competitive binding reaction, and the assay was then vortexed and incubated for $30 \mathrm{~min}$ at $4^{\circ} \mathrm{C}$. After the 30 min incubation period, the samples were centrifuged at $4^{\circ} \mathrm{C}$ at $3,500 \mathrm{rpm}$ for 15 minutes. The supernatants from the samples were decanted into scintillation vials, which were then filled with $3.5 \mathrm{~mL}$ of scintillation fluid (in each vial). The entire assay is then loaded into a Beckman liquid scintillation spectrometer (LS 1801) for final count and final values were calculated in Microsoft Excel ${ }^{\circledR}$.

A mixture of dried fecal samples were used to create a pooled sample and extracted by the above protocol. The resulting sample was diluted 2-fold from 1:2 to 1:256 in PBS and all dilutions run in the glucocorticoid assay. The displacement curve of glucocorticoid metabolites was parallel when compared to the standard curve $(r=0.0995, p<0.01)$. A known amount of exogenous corticosterone was added to pooled samples prior to sample extraction, and resulted in extraction of exogenous corticosterone of $102.8 \%$. A continuum of known amounts of exogenous corticosterone hormone $(n=8)$ was spiked with the sample matrix, resulting in $92.3 \% \pm 11.1 \%$ accuracy. Inter-assay coefficient of variation was $6.9 \%$ based on duplicates of high binding corticosterone controls and $7.2 \%$ on duplicates of low binding 
corticosterone controls. Intra-assay variation was found to be $6.60 \%$. Assay sensitivity was calculated to be $23.587 \mathrm{pg} /$ tube, which reflects $\% \mathrm{~B} / \mathrm{Bo}$ at $90 \%$ of our lowest standard.

The antibody used in this procedure was produced against corticosterone-3-carboxymethyloxime: BSA (ICN Biomedicals, Costa Mesa, CA). The antibody cross-reacts 100\% with corticosterone, 2.30\% with desoxycorticosterone, $0.47 \%$ with testosterone, $0.35 \%$ with prednisolone, $0.33 \%$ with $17 \alpha-$ hydroxyprogesterone, $0.27 \%$ with cortisol, $0.17 \%$ with progesterone, $0.14 \%$ with 11 -desoxycortisol, $0.07 \%$ with $20 \alpha$-dihydroprogesterone, and $0.05 \%$ with aldosterone.

\section{Keeper Surveys}

Surveys were distributed on June 1,2013 at the end of the behavioral data and fecal collection. Four keeper staff (i.e., raters) were given 18 personality trait adjectives and asked to rate each individual on each trait on a scale of one to ten (Wielebnowski, 1999). The amount of experience with cheetahs for each rater ranged from 3 to 25 years $(M=11.75, x \pm \mathrm{SD}=8.35)$.

\section{Statistical Analyses}

All behavioral data were corrected for percentage of time visible. Behaviors that were speciesspecific (i.e., pacing was not included) were calculated using the Shannon diversity index. The Shannon diversity index was used as it has the ability to detect subtle changes in diversity when one factor or in the current study, behavior, is dominant (DeJong, 1975; Shannon \& Weaver, 1949).

To determine the keepers' reliability on the surveys, Intra Class Correlation (ICC) was calculated using the ratings of each individual cheetah's personality traits. Based on previous studies, it was determined that ICC values $>0.600$ were indicative of good agreement between raters and any values below this were excluded from analysis (Baker \& Pullen, 2013; Shrout \& Fleiss; 1979). Personality traits with ICC values that deemed reliable $(>0.600)$, behaviors that were indicative of personality traits (Explore/Interact with Enrichment, Explore/Interact with Environment, Social Positive, Social Negative, Play) and FGM levels were entered for Principal Components Analysis (PCA). PCA orders the factors in relation to the proportion of the variance that these factors contribute to the dataset (Abdi, 2003). By utilizing an orthogonal rotation (varimax) method with Kaiser Normalization, the variance of each factor is maximized, producing uncorrelated factors that are simplified and interpretable for results (Abdi, 2003). All factors were clustered into five personality components, and each component was labeled based on the largest positive (and if applicable, negative) loading since larger factor loadings mean a greater contribution to that component (Stevenson-Hinde \& Zunz, 1978).

Based on non-normality and small sample size, nonparametric tests were utilized for all analysis. Spearman's rho correlation was used to examine associations between the personality components and indicators of welfare, which included behavioral diversity, pacing, self-maintenance, and other abnormal (Table 5). A Mann-Whitney U-test was used to examine associations of the personality components between gender, reproductively successful individuals, and hand-reared individuals. All data were analyzed using SPSS and alpha levels were set and considered significant at $p<0.05$.

\section{Results}

For inter-rater reliability of the surveys, ICC values for rater concordance ranged from 0.519 to 0.970 . Three personality traits from the survey were excluded due to low rater concordance $(<0.600)$ : calm (0.519), solitary (0.522), and vocal (0.552), and another three traits were excluded due to lack of data (friendly to conspecifics, fearful of conspecifics, tense). Table 3 displays ICC values for all personality traits and the 12 traits found to reach the criterion.

Based on the rotated component matrix, the five personality components attained from the data accounted for $88.0 \%$ of the variance. These five personality components from the 12 reliable traits were labeled Insecure, Aggressive, Interactive, Active, and Unsociable. There were significant correlations 
between the components and indicators of welfare. The components Aggressive and Interactive had a positive correlation with behavioral diversity, while Unsociable had a positive correlation with selfmaintenance (Table 5).

Table 3

\begin{tabular}{lc} 
Intra-Class Correlation (ICC) Values for All Personality Traits from Keeper Surveys & \\
\hline Trait & ICC Value \\
\hline Active & $\mathbf{0 . 7 7 5}$ \\
Aggressive to conspecifics & $\mathbf{0 . 8 3 8}$ \\
Aggressive to people & $\mathbf{0 . 9 0 7}$ \\
Calm & 0.519 \\
Curious & $\mathbf{0 . 7 8 7}$ \\
Eccentric & $\mathbf{0 . 6 6 3}$ \\
Excitable & $\mathbf{0 . 6 2 1}$ \\
Friendly to people & $\mathbf{0 . 9 7 0}$ \\
Fearful of people & $\mathbf{0 . 8 6 7}$ \\
Insecure & $\mathbf{0 . 8 3 2}$ \\
Playful & $\mathbf{0 . 8 3 7}$ \\
Self-assured & $\mathbf{0 . 8 8 4}$ \\
Smart & $\mathbf{0 . 8 9 5}$ \\
Solitary & 0.522 \\
Vocal & 0.522 \\
\hline
\end{tabular}

Note. Values used for analysis $(>0.600)$ are shown in boldface.

There were significant results regarding gender, reproductive success and hand-reared individuals (Table 6). Males scored higher than females on components Aggressive, $U(15)=64.0, p<0.05$, and Interactive, $U(15)=60.0, p<0.05$. Females that were hand-reared scored lower on Insecure, $U(7)=0.00$, $p<0.05$, and Aggressive, $U(7)=0.00, p<0.05$. Individuals with reproductive success scored higher on the component Unsociable, $U(15)=54.0, p<0.05$. Those with higher levels of the component Unsociable also had an association with high FGM levels (Table 4).

\section{Discussion}

The primary objective in this study was to apply a multifaceted method to assess personality of South African cheetahs at SDZSP. Utilizing the observer rating and coding methods in conjunction with hormone monitoring produced significant results. The five personality components derived from analysis were Insecure, Aggressive, Interactive, Active, and Unsociable. Although these components were not identical to previous personality research on cheetahs (Baker \& Pullen, 2013; Wielebnowski, 1999), they do share similarities with the components found in the Wielebnowski (1999) study based on factor loadings. Our first component Insecure closely resembles Tense-fearful in the Wielebnowski (1999) study, our third component Active resembles Vocal-Excitable, and our second component Aggressive matches with the third component of Wielebnowski (1999).

Differences between male and female subjects were expected due to behavioral differences observed in the wild (Caro, 1993). In this study, males displayed higher levels of the components Aggressive and Interactive. It was also found that individuals that scored higher on these components expressed higher levels of behavioral diversity. This difference was also observed in another solitary felid species, snow leopards. Gartner and Powell (2012) found that male snow leopards exhibited higher levels of activity, vigilance, and vocalization, which may suggest that males display more diverse behaviors than 
females. This also reflects what is seen in wild cheetahs, as males are known to have higher levels of exploratory behaviors to increase the probability of finding a female, and have higher levels of aggression due to competition with other males (Caro, 1993; Gartner \& Powell, 2012).

Table 4

Factor Loadings of Personality Component Analysis (PCA) with Varimax Rotation and Kaiser Normalization

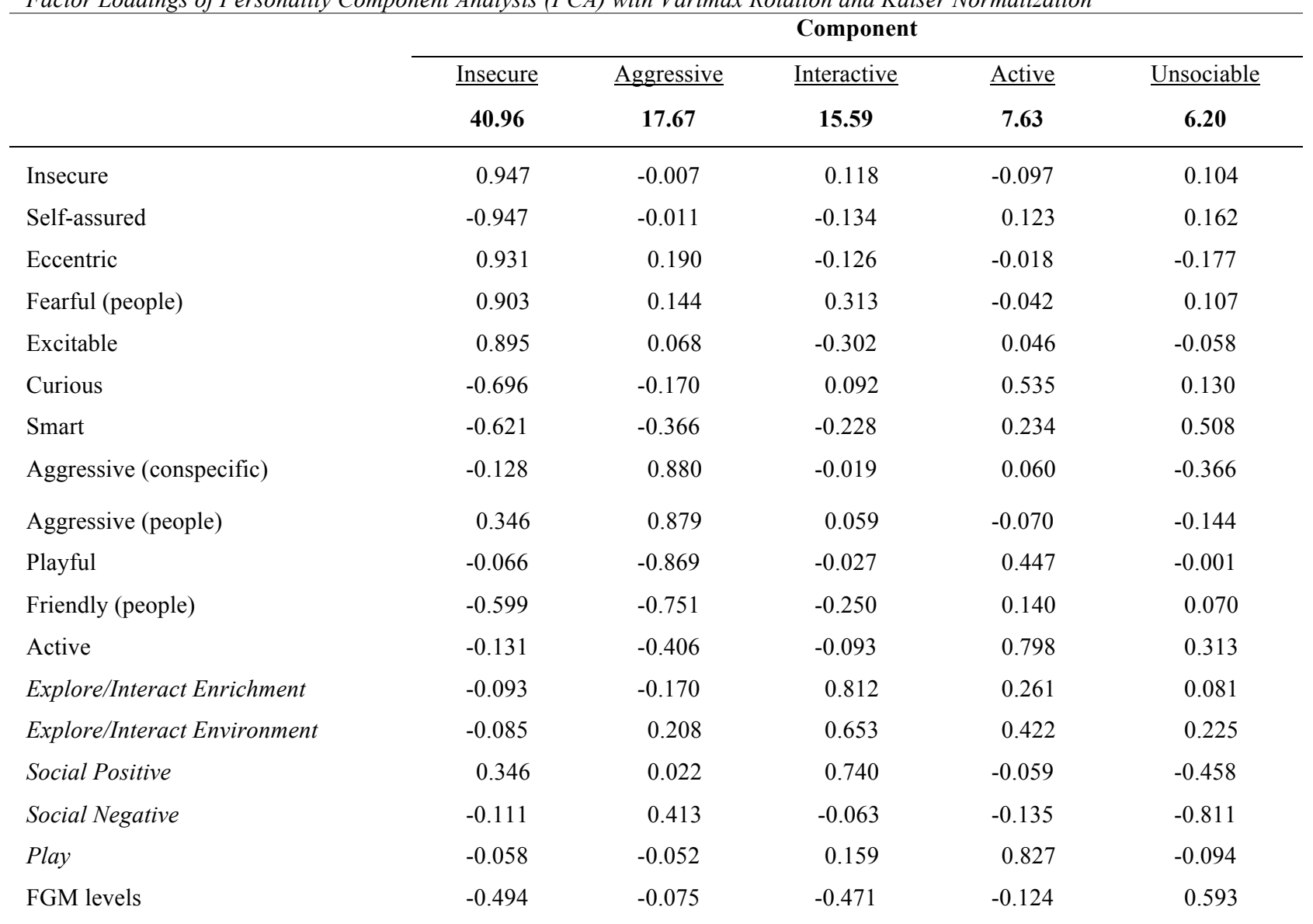

Note. \% variance explained is shown in boldface. Behavioral states are shown in italics. Rotation converged in 8 iterations.

Table 5

Spearman's Rho Correlation Coefficients of Personality Components Compared to Indicators of Welfare

\begin{tabular}{lccccc}
\hline & Insecure & Aggressive & Interactive & Active & Unsociable \\
\hline Pacing & -0.28 & 0.23 & -0.02 & 0.48 & 0.30 \\
Other Abnormal & -0.03 & 0.13 & 0.15 & -0.27 & -0.28 \\
Self-Maintenance & 0.14 & 0.22 & -0.14 & 0.25 & $0.81^{*}$ \\
Behavioral Diversity & 0.44 & $0.58^{*}$ & $0.64^{*}$ & 0.21 & 0.13 \\
\hline
\end{tabular}

${ }^{*} p<0.05$, two-tailed.

Individuals that were hand-reared were found to have lower scores on the personality components of Insecure and Aggressive. Similarly, in Wielebnowski (1999), rearing experience was investigated as a possible factor towards shaping personality and revealed that while not a significant result, hand-reared individuals displayed lower scores on Tense-fearful and Aggressive. Combined, these results suggest that hand-reared individuals are likely more comfortable with the keepers due to the difference in rearing. The 
raters could have been assessing that these individuals were more relaxed in their interactions with humans rather than their interactions with conspecifics, therefore having a lower score on insecure and aggressive traits (Kuhar et al., 2006; Mellen, 1991; Wielebnowski, 1999). Although the sample size of hand-reared individuals was small $(N=4)$, understanding the influences of different rearing techniques could aid in future management decisions regarding the species.

Table 6

Mann-Whitney U-Test Displaying Personality Components Compared with Gender, Reproductive Success, and Hand-Rearing

\begin{tabular}{|c|c|c|c|c|c|c|c|c|c|c|c|c|}
\hline & \multicolumn{4}{|c|}{ Gender } & \multicolumn{4}{|c|}{ Reproductive Success } & \multicolumn{4}{|c|}{ Hand-Rearing } \\
\hline & $U$ & $p$ & $\begin{array}{l}\text { MR } \\
(\mathrm{M})^{\mathrm{a}}\end{array}$ & $\operatorname{MR}(F)^{b}$ & $U$ & $p$ & $\begin{array}{l}\text { MR } \\
(W)^{c}\end{array}$ & $\begin{array}{c}\text { MR } \\
\left(\mathrm{Wo}^{\mathrm{d}}{ }^{\mathrm{c}}\right.\end{array}$ & $U$ & $p$ & $\begin{array}{l}\text { MR } \\
(Y)^{e}\end{array}$ & $\begin{array}{l}M R \\
(N)^{f}\end{array}$ \\
\hline Insecure & 31.0 & 0.67 & 8.38 & 9.56 & 21.0 & 0.38 & 7.20 & 9.75 & 0.0 & $0.02^{*}$ & 2.50 & 7.00 \\
\hline Aggressive & 64.0 & $0.01 *$ & 12.50 & 5.89 & 46.0 & 0.10 & 12.20 & 7.67 & 0.0 & $0.02 *$ & 2.50 & 7.00 \\
\hline Interactive & 60.0 & $0.02 *$ & 12.00 & 6.33 & 22.0 & 0.44 & 7.40 & 9.67 & 8.0 & 0.73 & 4.50 & 5.40 \\
\hline Active & 39.0 & 0.82 & 9.38 & 8.67 & 35.0 & 0.65 & 10.00 & 8.58 & 11.0 & 1.00 & 5.25 & 4.80 \\
\hline Unsociable & 32.0 & 0.74 & 8.50 & 9.44 & 54.0 & $0.01 *$ & 13.80 & 7.00 & 6.0 & 0.41 & 4.00 & 5.80 \\
\hline
\end{tabular}

Note. $\mathrm{MR}=$ mean rank.

${ }^{\mathrm{a}} \mathrm{M}=$ Males, $N=8 .{ }^{\mathrm{b}} \mathrm{F}=$ Females, $N=9 .{ }^{\mathrm{c}} \mathrm{W}=$ With reproductive success, $N=5 .{ }^{\mathrm{d}} \mathrm{Wo}=$ Without reproductive success, $N=12$.

${ }^{\mathrm{e}} \mathrm{Y}=$ Yes was hand-reared, $N=4 .{ }^{\mathrm{f}} \mathrm{N}=$ Not hand-reared, $N=5$.

${ }^{*} p<0.05$.

Animals that historically produced offspring were higher on the component Unsociable and on an indicator of welfare, self-maintenance. Being antisocial is natural for a cheetah since they are a solitary species and would not encounter many conspecifics in the wild, which is especially true for females (Caro, 1993). One study measured the impact of social factors on cheetahs in managed care and the authors found that ovarian suppression (i.e., decreased reproductive activity) is more prevalent in paired females in the same enclosure than separated females (Wielebnowski, Ziegler, Wildt, Lukas, \& Brown, 2002). Additionally, females that were paired displayed lower levels of self-grooming (i.e., selfmaintenance). Although similar to the results of the current study, comparisons between paired and separate females were not made but should be included in future research.

The results from the current study demonstrated that reproductively successful individuals that scored higher on the component Unsociable also displayed higher FGM levels. This result was inconsistent with previous findings as female cheetahs with high FGM values can be reproductively inactive and irregular in cycling (Jurke, Czekala, Lindburg, \& Millard, 1997; Wielebnowski et al., 2002). However, Jurke et al. (1997) analysis of metabolite measurement in cheetahs, one female demonstrated periods of increased ovarian activity while exhibiting high FGM levels at the same time. This particular female was described as having the highest anxiety levels in the study. The findings from Jurke et al. (1997) further suggest that individual behavioral differences are critical factors for understanding how personality affects reproductive success and how this can ultimately improve the management of breeding programs for cheetahs. Although our sample of individuals with reproductive success was small $(N=5)$ and reproductive success was not gender specific, the variances in our results compared to previous literature emphasize the importance of a multifaceted assessment of personality and context when examining animal personality.

Regarding personality research, numerous studies have demonstrated strong agreements between observer ratings and coding of behavior (Eckardt et al., 2014; Freeman, Schulte, \& Brown, 2010; Horback, Miller, \& Kuczaj, 2013). However, it could be noted that there may be certain limitations to the observer rating method. Some of the trait adjectives from the keeper survey were omitted from analysis due to low rater concordance or not having enough information to reach the criterion. One of the possibilities for these discrepancies could be due to the varying cheetah experience of the raters in this 
study (range $=3-25$ years). Another possibility is that the definitions of the traits on the survey were not clear to those who were not as experienced in rating behavior. Although inter-rater reliability was deemed reliable, timing can also be a concern as keepers often have limited time to directly observe animals for elongated periods and may miss observing infrequent occurrences of behavior (Freeman et al., 2010; Margulis \& Westhus, 2008). In order to have more consistent results, future research could implement having raters with a certain length of experience with the study animals (e.g., more than 5 years) and with using the rating method to reduce the possibility of a rater not having the same amount of knowledge as the other raters.

Despite its challenges, observer ratings ultimately exploit the intimate knowledge of those that are the most familiar with individuals, and are a valuable resource for understanding subtle changes in behavior that can determine individuality (Carlstead, Mellen, et al., 1999). Since personality is defined as observing behavioral differences consistently over time and across all contexts, then future research should integrate the advantageous observer rating method as part of a multifaceted approach in order to demonstrate stability (Gosling, 2001; Meagher, 2009). This study displayed that using the combination of multiple methods provides greater insight into understanding how an individual responds to the environment, which could assist zoological institutions in future management decisions. Although this study had significant results regarding reproductive success, further research using a larger sample size is needed to understand how individual behavioral differences ultimately impact reproduction, especially for a species that is not self-sustaining in professional care. By demonstrating that personality research has immense potential to impact how animals are professionally managed in zoological facilities, future application of this research has the possibility of increasing sustainability in managed populations.

\section{Acknowledgements}

We would like to thank Dr. Allison Alberts, Randy Rieches, and Dr. Matt Anderson for their continued support. We would like to thank Megan McMahon, Emily Steward, and Michelle Wechsler for all of their assistance in collecting behavioral data and sample processing. Finally, we would like to thank Autumn Nelson and the entire cheetah care staff at the San Diego Zoo Safari Park for making this project possible. This research was undertaken with a grant from the Institute of Museum and Library Services (IMLS).

\section{References}

Abdi, H. (2003). Factor rotations in factor analyses. In A. Bryman, T. Futing, \& M. Lewis-Beck (Eds.), Encyclopedia of social sciences research methods. Retrieved from http://www.utdallas.edu/ herve/Abdi-rotations-pretty.pdf

Baker, K., \& Pullen, K. P. (2013). The impact of housing and husbandry on the personality of cheetah (Acinonyx jubatus). Journal of Zoo and Aquarium Research, 1, 35-40.

Brown, J. L., Wildt, D. E., Wielebnowski, N., Goodrowe, K. L., Graham, L. H., Wells, S., \& Howard, J. G. (1996). Reproductive activity in captive female cheetahs (Acinonyx jubatus) assessed by fecal steroids. Journal of Reproduction and Fertility, 106, 337-346.

Carlstead, K., Fraser, J., Bennett, C., \& Kleiman, D. G. (1999). Black rhinocerous (Diceros bicornis) in U.S. zoos II: Behavior, breeding success and mortality in relation to housing facilities. Zoo Biology, 18, 35-52.

Carlstead, K., Mellen, J., \& Kleiman, D. G. (1999). Black rhinoceros (Diceros bicornis) in U.S. zoos I: Individual behavior profiles and their relationship to breeding success. Zoo Biology, 18, 17-34.

Caro, T. M. (1993). Behavioral solutions to breeding cheetahs in captivity: Insights from the wild. Zoo Biology, 12, $19-30$.

DeJong, T. M. (1975). A comparison of three diversity indices based on their components of richness and evenness. Oikos, 26, 222-227.

Eckardt, W., Steklis, H. D., Steklis, N. G., Fletcher, A. W., Stoinski, T. S., \& Weiss, A. (2015). Personality dimensions and their behavioral correlates in wild Virunga mountain gorillas (Gorilla beringei beringei). Journal of Comparative Psychology, 129, 26-41.

Freeman, H. D., \& Gosling, S. D. (2010). Personality in nonhuman primates: A review and evaluation of past 
research. American Journal of Primatology, 72, 653-671.

Freeman, E. W., Schulte, B. A., \& Brown, J. L. (2010). Using behavioral observations and keeper questionnaires to assess social relationships among captive female African elephants. Zoo Biology, 29, 140-153.

Gartner, C. M., \& Powell, D. (2012). Personality assessment in snow leopards. Zoo Biology, 31, 151-165.

Gosling, S. D. (2001). From mice to men: What can we learn about personality from animal research? Psychological Bulletin, 127, 45-86.

Highfill, L., Hanbury, D., Kristiansen, R., Kuczaj, S., \& Watson, S. (2010). Rating vs. coding in animal personality research. Zoo Biology, 29, 509-516.

Horback, K. M., Miller, L. J., \& Kuczaj, S. A. (2013). Personality assessment in African elephants (Loxodonta africana): Comparing the temporal stability of ethological coding versus trait rating. Applied Animal Behaviour Science, 149, 55-62.

Jurke, M. H., Czekala, N. M., Lindburg, D. G., \& Millard, S. E. (1997). Fecal corticoid metabolite measurement in the cheetah (Acinonyx jubatus). Zoo Biology, 16, 133-147.

Kuhar, C. W., Stoinski, T. S., Lukas, K. E., \& Maple, T. L. (2006). Gorilla behavior index revisited: Age, housing, and behavior. Applied Animal Behaviour Science, 96, 315-326.

Laurenson, M. K., Caro, T., \& Borner, M. (1992). Female cheetah reproduction. National Geographic Research \& Exploration, 8(1), 64-75.

Margulis, S. W., \& Westhus, E. J. (2008). Evaluation of different observational sampling regimes for use in zoological parks. Applied Animal Behaviour Science, 110, 363-376.

Marker, L. (2013). International cheetah (Acinonyx jubatus) studbook. Otjiwarongo, Namibia: Cheetah Conservation Fund.

Marker, L., \& O'Brien, S. (1989). Captive breeding of the cheetah (Acinonyx jubatus) in North American zoos (1871-1986). Zoo Biology, 8, 3-16.

Marker-Kraus, L., \& Kraus, D. (1997). Conservation strategies for the long-term survival of the cheetah. International Zoo Yearbook, 35, 59-66.

Meagher, R. K. (2009). Observer ratings: Validity and value as a tool for animal welfare research. Applied Animal Behaviour Science, 119, 1-14.

Mellen, J. D. (1991). Factors influencing reproductive success in small captive exotic felids (Felis spp.): A multiple regression analysis. Zoo Biology, 10, 95-110.

Powell, D. M., \& Gartner, M. C. (2011). Applications of personality to the management and conservation of nonhuman animals. In M. Inoue-Murayama, S. Kawamura, \& A. Weiss (Eds.), From genes to animal behavior (pp. 185-199). Japan: Springer.

Shannon, C. E., \& Weaver, W. (1949). The mathematical theory of communication. Urbana, IL: University of Illinois Press.

Shrout, P. E., \& Fleiss, J. L. (1979). Intraclass correlations: Uses in assessing rater reliability. Psychological Bulletin, 86, 420-428.

Stevenson-Hinde, J., \& Zunz, M. (1978). Subjective assessment of individual rhesus monkeys. Primates, 19, 473482.

Vazire, S., \& Gosling, S. D. (2004). Personality and temperament: A comparative perspective. In M. Bekoff (Ed.), Encyclopedia of animal behavior (pp. 818-822). Westport, CT: Greenwood Publishing Group.

Watters, J. V., \& Powell, D. M. (2011). Measuring animal personality for use in population management in zoos: Suggested methods and rationale. Zoo Biology, 29, 1-12.

Wielebnowski, N. C. (1999). Behavioral difference as predictors of breeding status in captive cheetahs. Zoo Biology, $18,335-349$.

Wielebnowski, N. C., Ziegler, K., Wildt, D. E., Lukas, J., \& Brown, J. L. (2002). Impact of social management on reproductive, adrenal and behavioral activity in the cheetah (Acinonyx jubatus). Animal Conservation, 5, 291-301. 